\title{
Ville et campagne dans (et autour de) Nouvelles de Nulle Part
}

Town and Country in (and around) News from Nowhere

\section{Emmanuel Roudaut}

\section{(2) OpenEdition}

1 Journals

\section{Édition électronique}

URL : http://journals.openedition.org/rfcb/3261

DOI : 10.4000/rfcb.3261

ISSN : 2429-4373

\section{Éditeur}

CRECIB - Centre de recherche et d'études en civilisation britannique

\section{Édition imprimée}

Date de publication : 1 septembre 2004

ISBN : 2-911580-19-2

ISSN : 0248-9015

\section{Référence électronique}

Emmanuel Roudaut, "Ville et campagne dans (et autour de) Nouvelles de Nulle Part », Revue Française de Civilisation Britannique [En ligne], XIII-1 | 2004, mis en ligne le 01 septembre 2004, consulté le 07 octobre 2019. URL : http://journals.openedition.org/rfcb/3261 ; DOI : 10.4000/rfcb.3261

Ce document a été généré automatiquement le 7 octobre 2019.

\section{cc)}

Revue française de civilisation britannique est mis à disposition selon les termes de la licence Creative Commons Attribution - Pas d'Utilisation Commerciale - Pas de Modification 4.0 International. 


\title{
Ville et campagne dans (et autour de) Nouvelles de Nulle Part
}

\author{
Town and Country in (and around) News from Nowhere
}

\author{
Emmanuel Roudaut
}

1 Remontant le cours de la Tamise à la découverte de la société communiste future, le narrateur de Nouvelles de nulle part semble à bien des égards remonter le cours du temps vers un Âge d'or. Quant au paradis fugacement retrouvé où il entrevoit le bonheur avec la jeune Ellen, il a les traits idylliques d'un rêve rural dont le cadre évoque celui de l'Angleterre préindustrielle. Dans l'utopie de Morris, l'anticipation se mêle donc aux images d'un "temps d'avant », en dépit du marxisme de son auteur. En outre, si l'un des objectifs de Marx et Engels, qui préconisaient des «mesures tendant à supprimer progressivement l'opposition ville-campagne $»^{1}$ semble atteint dans Nouvelles de Nulle Part, c'est d'une manière singulière et peu conforme aux vues de Marx et Engels sur l'«abrutissement de la vie des champs $»^{2}$. Plus encore que dans un Londres régénéré et presque ruralisé, c'est au village et par le travail des champs que l'idéal communiste de Morris trouve son accomplissement.

2 Le modèle de société prôné par Morris puise donc à des sources diverses et contrastées, qui ne se limitent pas aux figures tutélaires de Carlyle, Marx, More et Ruskin. On s'attachera ici à décrire les formes particulières que revêt la synthèse entre ville et campagne imaginée par Morris avant d'évoquer les sources et la postérité de son ruralisme.

\section{Métamorphose de la ville}

3 Au début du roman, le personnage principal, dont le narrateur se fera le double dès le deuxième chapitre, regagne son domicile de Hammersmith à l'issue d'une réunion de la Socialist League fort semblable à celles de la section de William Morris ${ }^{3}$. Cela donne lieu à une évocation du quartier où vivait l'auteur. Le Londres de la fin de l'époque victorienne $e^{4}$ est décrit dans les termes les plus noirs. La laideur oppressante qui 
caractérise les innovations technologiques de la civilisation de la machine (pont métallique suspendu, métro) contraste fortement avec la beauté du monde où son rêvevision va le transporter ${ }^{5}$. Cette opposition manichéenne entre le Londres contemporain et celui des siècles à venir est renforcée par le changement de saison et la transition d'une scène nocturne à une scène matinale. Transporté dans l'avenir, le narrateur découvre un Hammersmith méconnaissable, car lavé de toutes les souillures de la civilisation urbaine, à l'instar des environs (Chiswick, Barn Elms) et de la Tamise où l'on peut à nouveau se baigner et pêcher le saumon. Le jeune batelier qui lui sert de guide, Dick Hammond, déconcerté par la surprise de l'« étranger » et ses questions, décide de le présenter à son arrière-grand-père, le vieux Hammond, dont les connaissances historiques pourront satisfaire sa curiosité. Le trajet vers le domicile du vieil homme, qui s'est installé dans les locaux de l'ancien British Museum, à Bloomsbury, occupe plusieurs chapitres (4 à 8) et va étoffer la description du Londres des temps nouveaux esquissée par celle de Hammersmith. Mais alors que l'on quitte l'ancien «faubourg miteux » (shabby suburb) pour traverser les quartiers huppés de l'ouest et atteindre le centre de la capitale, l'impression initiale se confirme. La métropole a perdu ses attributs pour se muer en une série de bourgades séparées par la forêt. L'un des nombreux indices de cette mutation topographique est la disparition du tracé des rues. Le terme même de rue (street), dont les connotations sont trop urbaines, n'a plus cours ; on ne parle plus que de route (road) ou de chemin (highway). Le quartier de Trafalgar Square est logé à la même enseigne que l'ancienne King Street de Hammersmith et qu'Endell Street. Whitehall, haut lieu du pouvoir s'il en fut, a disparu des mémoires pour n'être plus qu'une voie reliant un verger (l'ancien Trafalgar Square) au « marché au fumier » (l'ancien parlement) ${ }^{6}$.

4 Le parcours dans Londres s'arrête au British Museum, mais la description de ce qui fut l'East End et la City figure dans le chapitre 10, insérée dans le long récit où le vieux Hammond explique à son visiteur le déroulement de la révolution et le passage à la société communiste. La City, l'ancien quartier d'affaires, est la seule zone bâtie à présenter encore une importante densité de population, ce qui constitue non pas une survivance, mais une inversion par rapport à ce qui prévalait à la fin de l'époque victorienne. Les immeubles luxueux autrefois désertés après les heures de travail sont revenus à un usage d'habitation ${ }^{7}$. En revanche, les taudis notoirement surpeuplés de l' East End ont disparu et cédé la place à un habitat plus dispersé dans un paysage campagnard. Les commémorations de l'éradication de ces poches de misère (the Clearing of Misery), célébrées chaque année le jour du Premier Mai, présentent tous les aspects d'une fête villageoise et l'ancienne réalité qu'elles évoquent est inconcevable pour les participants ${ }^{8}$.

5 En fait, le Londres que connaissait le narrateur ('the brick and mortar desert of London') s'est dissous dans la campagne environnante, tout juste a-t-il laissé un peu plus de traces de son existence que les grands centres industriels ${ }^{10}$ dont la croissance avait été exponentielle au dix-neuvième siècle. Toute trace de l'époque victorienne semble avoir disparu, non seulement physiquement, mais aussi dans le souvenir des hommes. Ainsi, lorsque Dick évoque le passé de Londres devant le visiteur, ses références littéraires et historiques s'arrêtent à Shakespeare et à l'époque élisabéthaine ${ }^{11}$. La grande ville a donc été condamnée à disparaître dans le monde nouveau. La seule survivance urbaine de l'ancien monde est la ville aux dimensions plus modestes, elle seule a conservé sa topographie distinctive, avec ses rues et ses places ${ }^{12}$. Oxford, où Morris a été étudiant, 
est citée en exemple, car même au dix-neuvième siècle, elle avait su préserver l'architecture "précommerciale ", mais elle ne fait plus figure d'exception. D'autres villes moyennes, trop défigurées par le dix-neuvième siècle, ont été reconstruites dans le même esprit ${ }^{13}$.

6 Loin d'être futuriste, la ville utopique de William Morris évoque donc fortement l'Angleterre d'avant la révolution industrielle. Elle est avant tout d'inspiration médiévale, à tel point que le narrateur a l'impression d'être revenu au quatorzième siècle :

There were houses about, some on the road, some amongst the fields with pleasant lanes leading down to them, and each surrounded by a teeming garden. They were all pretty in design, and as solid as might be, but countrified in appearance, like yeomen's dwellings; some of them of red brick like those by the river, but more of timber and plaster, which were by the necessity of their construction so like mediaeval houses of the same material that I fairly felt as if I were alive in the fourteenth century. ${ }^{14}$

7 La référence au Moyen Âge est également perceptible dans de multiples considérations vestimentaires et architecturales qui sortiraient du cadre de cet article, mais son premier aspect est le souvenir d'une époque où la ville et la campagne s'interpénétraient, comme le précise Morris dans une de ses conférences : 'On the whole, then, the towns of the Middle Ages, in this country at least, were a part of the countrysides where they stood. ${ }^{15}$

\section{Exaltation de la campagne}

De retour à Hammersmith après sa longue entrevue avec le vieux Hammond, le narrateur entreprend un nouveau voyage qui revêt presque une dimension initiatique car il peut enfin se dépouiller de ses habits victoriens pour endosser les beaux vêtements de l'ère nouvelle. Il s'agit d'accompagner son guide, Dick, et l'épouse de celui-ci, Clara, aux environs d'Oxford, afin de participer aux travaux de la fenaison qui seront l'occasion d'une grande fête.

9 À partir du chapitre 21, le récit est entièrement consacré à cette remontée de la Tamise en barque, sur un trajet dont Morris était familier ${ }^{16}$. Cette fois, l'itinéraire emprunté ne traverse plus Londres, ou ce qu'il en reste, mais ce qui était une Angleterre périurbaine et rurale à la fin de l'époque victorienne. Les villas 'cockney', c'est-à-dire édifiées récemment par des nouveaux riches, ont disparu des bords de la Tamise et il s'avère qu'un terme a été mis à l'étalement urbain anarchique du dix-neuvième siècle ${ }^{17}$, au grand soulagement du narrateur, double de Morris, qui avait déjà fait allusion à ce problème en évoquant son enfance près de la forêt d'Epping ${ }^{18}$. Le cas d'Epping, situé au nord-est de Londres, à proximité de l'East End, est emblématique du conflit entre la spéculation immobilière et l'accès des couches populaires aux loisirs de plein air. Faisant fi du droit coutumier et au prix d'affrontements répétés avec les riverains et usagers, les propriétaires avaient tenté d'interdire l'accès de la forêt au public avant d'être déboutés en justice en 1874, ce qui avait permis aux édiles de la Cité de Londres d'en faire l'acquisition afin d'en garantir le libre accès et de prévenir toute tentative de lotissement ${ }^{19}$. Une menace analogue sur Wimbledon Common est à l'origine de la création en 1865 d'un groupe de pression encore actif de nos jours, la Commons, Open Spaces and Footpaths Preservation Society ${ }^{20}$, qui avait notamment pour objectif de 
s'opposer au lotissement abusif de commons ${ }^{21}$ et de garantir les droits de passage sur les chemins et sentiers.

Quand il s'extasie sur l'abondance d'oiseaux autour de lui, le narrateur fait indirectement allusion à une autre forme de privatisation (au dix-neuvième siècle) d'un espace rural précédemment accessible à tous. "L'époque des gardes-chasse est révolue ", constate-t-i ${ }^{22}$. Certes, Morris le socialiste prend pour cible une profession au service des possédants et la répression du braconnage a toujours été vilipendée comme le symbole des privilèges abusifs de la caste nobiliaire, mais ici Morris l'écologiste vise surtout la vogue toute récente de la battue, qui se traduit par des hécatombes de coqs de bruyère ou de faisans ${ }^{23}$. Il met alors le doigt sur un phénomène en plein essor, celui des locations de chasse, qui permet aux propriétaires fonciers de compenser la chute des loyers et fermages provoquée par la grande crise de l'agriculture (circa 1875-1895). Les landes, notamment celles d'Écosse et du Derbyshire, seront ainsi louées (fort cher) à des chasseurs fortunés, tandis que les éleveurs d'ovins en seront écartés, de même que les randonneurs. Les gardes-chasse, dont les effectifs ne cessent de croître à l'époque, sont les agents d'une politique d'exclusion qui deviendra un symbole politique dans l'entre-deux-guerres ${ }^{24}$.

11 L'une des conséquences du marasme agricole fut la baisse de la démographie rurale, ce qui constituait une nouveauté. Jusqu'aux années 1880 , l'excédent de la population des campagnes avait suffi à alimenter l'expansion urbaine. Désormais, l'exode rural menaçait de vider un réservoir de main-d'œuvre "saine ", seule susceptible, aux yeux des tenants du darwinisme social, de contenir la dégénérescence à laquelle la population ouvrière urbaine était condamnée du fait de son environnement ${ }^{25}$. Dans Nouvelles de Nulle Part, le dépeuplement des campagnes britanniques à la fin du dixneuvième siècle est bien évoqué par le vieux Hammond ${ }^{26}$, mais un mouvement migratoire inversé lui a succédé. La ville a envahi la campagne, mais il ne s'agit pas d'une urbanisation à marche forcée comme celle qui sévissait aux abords des grandes villes et qui avait englouti Walthamstow, le village natal de Morris. Il s'agit au contraire d'une « ruralisation » des populations citadines, dont le trajet vers Bloomsbury a donné

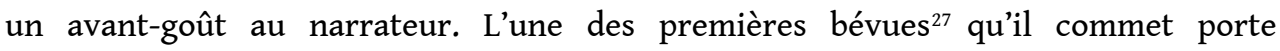
précisément sur ce sujet. Le terme de "campagnard» (country people) qu'il utilise au marché d'Hammersmith n'a aucun sens pour Dick, tout simplement parce que la distinction entre ville et campagne n'a plus lieu d'être. S'il subsiste une différence qualitative, elle semble plutôt favorable aux habitants des contrées les plus sauvages et les plus reculées, au dire du jeune homme :

There are parts of these islands which are rougher and rainier than we are here, and there people are rougher in their dress; and they themselves are tougher and more hard-bitten than we are to look. But some people like their looks better than ours; they say they have more character in them - that's the word. Well, it's a matter of taste. - Anyhow, the cross between us and them generally turns out well.

28

Certes, l'interpénétration entre ville et campagne dans l'Angleterre du XXII ${ }^{\mathrm{e}}$ siècle peut se lire comme la réalisation du souhait de Marx et Engels et la participation des « citadins » aux travaux des champs marque la fin de la séparation entre «l'animal des villes et celui des campagnes ", "base fondamentale " de la division du travail dont Marx déplorait les effets dans Le Capital ${ }^{29}$. En outre, certains propos du vieux Hammond semblent souligner l'effet bénéfique de l'apport citadin à cette synthèse qui aurait 
permis à la population campagnarde de s'arracher à «l'abrutissement de la vie des champs »:

The difference between town and country grew less and less, and it was indeed this world of the country vivified by the thought and briskness of town-bred folk which has produced this happy and leisurely but eager life of which you have had a first taste. $^{30}$

Il reste que l'agent principal de cette synthèse demeure le monde campagnard ('this world of the country...has produced'), la ville n'a été que l'instrument auxiliaire de la transformation dont, en revanche, elle a été le principal objet :

The town invaded the country; but the invaders, like the warlike invaders of early days, yielded to the influence of their surroundings, and became country people; and in their turn, as they became more numerous than the townsmen, influenced them also. ${ }^{31}$

14 En fait, s'il semble s'être opéré une fusion entre monde rural et monde urbain dans l'Angleterre du XXII ${ }^{e}$ siècle, c'est au prix d'un renversement des codes dictés par Londres qui prévalaient à l'époque victorienne. Un exemple en est fourni par les nouveaux canons de la beauté féminine, illustrés par Clara et Ellen, la jeune fille chez qui le groupe fait étape en remontant la Tamise. Alors que le grand-père d'Ellen, un " grincheux » nostalgique de l'ancien temps, déplore la sobriété de sa tenue et surtout son teint hâlé par les travaux des champs, ceux-ci font l'envie de la Londonienne qu'est Clara $^{32}$. D'autres épisodes laissent entendre que dans la société nouvelle, la simplicité rustique et le bronzage sont devenus un atout de séduction. Le temps des ombrelles est révolu ${ }^{33}$, c'est désormais le hâle paysan qui donne le ton. Il est toutefois précisé que si la majorité de la population a adopté un mode de vie rural, celui-ci n'a plus rien à voir avec l'abrutissement raillé par Marx ${ }^{34}$. L'apathie d'antan, le manque d'intérêt pour l'environnement dans la campagne victorienne « cockneyisée » ont cédé la place à une curiosité générale pour la faune et la flore ${ }^{35}$. La campagne victorienne ('an ill-kept, poverty-stricken farm, pillaged by the masters of the workshops ${ }^{36}$ ) s'est transformée en jardin. Ont disparu aussi bien la misère liée à la crise agricole que la vulgarité importée de la ville. Si mutation il y a eu, il s'agit d'un retour à une ruralité plus authentique. On retrouve là les préoccupations de l'auteur, qui flétrissait les goûts de ses contemporains en matière de résidences rurales :

I am thinking of the result of the residence of rich men in the countryside. The squalor of the farmers above mentioned is no doubt largely the result of stupidity, but with them poverty can be pleaded; but the rich squire-archy and nobility are not forced by lack of pence to cockneyize the countryside; but yet so soon as ever you come across a village turned smart but dull by idiotic architect-tooral-looral excrescences and changes; the cock-tailed school, the restored church, the Lady Bountiful cottages, the lodges of the Bayswater pattern, the carpet gardening of the vicar's garden and so forth, then look out for the big house of my lord or Sir Robert, or Captain Killmister, and you are sure to find it presently and unless it be an old house, or you have not got the real use of your eyes, you will be heartily sorry that you haven't missed it, such a lump of ugliness and vulgarity it will be. ${ }^{37}$

15 C'est au terme de sa progression dans la "vraie » campagne anglaise que le narrateur éprouve le sentiment d'avoir enfin découvert la société communiste dont les membres, en se dépouillant de leurs possessions, ont atteint le bonheur et la véritable richesse ${ }^{38}$. L'apothéose attendue est la fête des fenaisons ${ }^{39}$, qui doit se tenir dans l'ancienne église du village et dont les fêtes commémoratives des Great Clearings de l'East End étaient largement inspirées. Ce sont les rites campagnards qui servent de modèle à la ville et 
non plus le style de Bayswater qui s'exporte aux champs. Si symbiose entre ville et campagne il y a, elle se traduit par une suprématie des valeurs de la campagne. C'est par le primat de la ruralité que la société communiste se distingue de la société capitaliste où la ville exerçait son hégémonie :

The country, which, instead of being the due fellow and helpmate of the towns and the Town, is a troublesome appendage, an awkward incident of town life, which, commercial or intellectual, is the real life of our epoch. ${ }^{40}$

Même au fond de Bloomsbury, quand le vieux Hammond tente d'expliquer au visiteur l'inutilité des partis politiques dans le monde nouveau, il décrit une société dont les principales préoccupations tournent autour des travaux des champs ${ }^{41}$. Quant à Dick, son intérêt fréquemment exprimé pour le rythme des saisons et des jours ${ }^{42}$ surprend le Victorien transplanté par sa simplicité villageoise qu'il juge enfantine.

De même, Ellen proclame son amour de la terre et des choses de la nature, et le narrateur perçoit le contraste entre cette joie de vivre terrienne et l'aridité des intellectuels de son époque, coupés des réalités matérielles. Londres existe encore, mais elle n'est plus le centre, ses valeurs médiocres et vulgaires et sa civilisation du pis-aller (makeshift) ne rayonnent plus; au contraire, c'est elle qui est devenue un appendice de la campagne triomphante.

\section{Un socialisme rural ?}

Si la ville n'a pas disparu dans la société communiste imaginée par Morris, la campagne $\mathrm{y}$ occupe donc une place centrale et la nature y constitue la référence suprême, à tel point que l'on a pu y voir l'expression d'un «socialisme rural » qui s'opposerait au « socialisme urbain » de Bellamy. On sait que la publication en 1887 de Looking Backward par cet auteur américain était l'une des raisons qui avaient poussé Morris à rédiger Nouvelles de Nulle Part, en réaction à la société communiste proposée dans l'utopie de Bellamy, trop enrégimentée à ses yeux. À l'évidence, le caractère trop urbanisé du modèle proposé constituait l'un des principaux griefs de Morris :

Mr Bellamy's ideas of life are curiously limited; he has no idea beyond existence in a great city; his dwelling of man in the future is Boston (USA) beautified. In one passage, indeed, he mentions villages, but with unconscious simplicity shows that they do not come into his scheme of economic equality, but are mere servants of the great centres of civilization. This seems strange to some of us, who cannot help thinking that our experience ought to have taught us that such aggregations of population afford the worst possible form of dwelling-place, whatever the secondworst might be. ${ }^{43}$

L'étiquette de «socialisme rural » est pourtant fermement récusée par Meier. Celui-ci déclare que dans cette "fusion harmonieuse, ... il serait faux de penser qu'une prédominance excessive est accordée [au paysage de la campagne sur celui de la ville] $»^{44}$. S'efforçant de démontrer que la civilisation urbaine n'a pas disparu dans la vision morrissienne, Meier ${ }^{45}$ fait grand cas de la densité de peuplement qui prévaut dans la City et souligne que l'on continue à construire dans ce qui fut Long Acre (début du chapitre 8). Il omet cependant de préciser qu'il s'agit de maisons basses qui n'ont plus grand-chose en commun avec l'environnement du quartier en 1890.

Quant au ton employé par Dick à cette occasion, il montre bien qu'il s'agit d'un phénomène très inhabituel pour lui et ses contemporains. S'appuyant sur un autre propos ambigu du narrateur ${ }^{46}$, Meier ajoute que Londres, quoique transfiguré, "est 
toujours une grande ville, une capitale et même 'un centre intellectuel' ». Force est pourtant de constater que les connaissances livresques semblent bien dévaluées dans l'Angleterre du XXII e siècle.

21 Le primat du travail manuel, jugé plus gratifiant, fait qu'elles sont simplement tolérées comme une aimable excentricité ${ }^{47}$ et c'est en toute humilité que les érudits viennent prendre leur part aux travaux de la fenaison. Si l'adhésion du narrateur à cette évolution n'est pas totale, un écrit de Morris presque contemporain de la rédaction de Nouvelles de Nulle Part est sans ambiguïté. Après avoir déploré l'enlaidissement de la campagne sous le système capitaliste, voici ce qu'il précise :

Let me turn the leaf and find a new picture, or my holiday is spoilt ; and don't let some of my Socialist friends with whom I have wrangled about the horrors of London say, 'This is all that can come of your country life'. For as the round of the seasons under our system of landlord farmer and labourer produces in the country pinching parsimony and dulness [sic], so does the 'excitement of intellectual life' in the cities produce the slum under the capitalist system of turning out and selling market wares not for use but for waste. Turn the page I say. ${ }^{48}$

Si Meier prend acte des nombreuses diatribes anti-urbaines de Morris, c'est pour en minimiser la portée, notamment dans Nouvelles de Nulle Part comme on l'a vu. Pour ce faire, il s'appuie tout particulièrement sur des propositions d'urbanisme rédigées avec Bax en $1893^{49}$. Trois systèmes y sont envisagés, dont l'un laisserait subsister les grandes villes. Il apparaît toutefois clairement que ce n'est pas celui qui a la préférence de Morris, et l'embarras de Meier persiste face à certains aspects de son utopie. La fusion totale des modes de vie campagnard et urbain dans la société communiste est ainsi taxée d' "impétuosité peu réaliste "50. Meier tente d'expliquer cette "erreur» en l'historicisant : l'étalement industriel aurait été, à l'époque victorienne, "plus choquant qu'aujourd'hui", puisque l'électrification a eu raison "des nuages de fumée et des souillures de suie ». Les problèmes des grandes villes auraient été "plus ou moins palliés par le lent progrès des luttes syndicales et les progrès de la science ${ }^{51}$. Le propos implicite est que s'il avait connu les progrès réalisés au $\mathrm{XX}^{\mathrm{e}}$ siècle, Morris aurait modéré son ardeur antiurbaine teintée de babouvisme. Toutefois, l'identification de nouvelles formes de pollution, moins visibles, mais tout aussi dangereuses que les suies victoriennes, et plus encore l'explosion planétaire de la démographie urbaine depuis la publication de la thèse de Meier en 1971 remettent fortement en cause le tableau optimiste du devenir urbain brossé par celui-ci.

Sur ce point précis, l'interprétation de l'immense spécialiste de Morris qu'est Meier semble contestable; elle illustre en revanche le "scientisme urbanophile" d'une certaine orthodoxie marxiste. En outre, la société sans classes décrite par le vieux Hammond est fortement décentralisée, toute idée d'État-nation a bien entendu disparu et l'on y pratique une démocratie locale directe, à l'échelle de la bourgade ou de la paroisse $^{52}$. Le tableau ainsi dressé aurait pu recevoir l'assentiment des anarchistes, et l'on sait que Morris s'opposait moins à eux sur les objectifs que sur la stratégie à adopter. En revanche, il est peu probable que ses vues eussent été conciliables avec les théories de Lénine, qui préconisait le maintien d'un État fort pendant le premier stade de la société sans classes ${ }^{53}$. Toujours est-il que dans la vision utopique de Morris, non seulement le dépérissement de l'État doit intervenir le plus rapidement possible, mais il semble incompatible avec la survivance de grands centres urbains ${ }^{54}$. 


\section{Une révulsion très victorienne}

24 La mise en accusation de la ville, omniprésente dans les essais et conférences de Morris comme dans ses textes de fiction, ne prend pas seulement pour cible la pollution et les souillures de la civilisation industrielle. Plus encore que les cités manufacturières, vouées à disparaitre puisque totalement dépourvues de lien avec le passé médiéval, c'est Londres qui fait l'objet des attaques les plus cinglantes sous la plume de Morris :

There is, indeed, ... something soul-deadening and discouraging in the ugliness of London; other ugly cities may be rougher and more savage in their brutality, but none are so desperately shabby, so irredeemably vulgar as London. It is difficult to express in words the feelings with which this 'cockney nightmare' burdens me... Rich London is the creature of slum-London, of poor London; and though I do not say that the London slums are worse than those of other big cities, yet they together with the rich quarters make up the monstrosity we call London, which is at once the centre and the token of the slavery of commercialism which has taken the place of the slaveries of the past. ${ }^{55}$

Ailleurs, c'est à l'emprise croissante de la capitale sur la vie de la nation qu'il attribue en premier lieu la coupure fatidique entre vie urbaine et vie rurale :

In the Middle Ages even London was no more of a centre than Bristol or York... But in the eighteenth century London was become [sic] very decidedly the centre of England, and now the distinction was not between the town and the countrysides, but between London and the rest of the country, towns and all. And here properly begins the opposition of town to country. The only other further development of this was the work of the Great Industries which created the big manufacturing town. ${ }^{56}$

Cette focalisation sur Londres est caractéristique de la fin de l'époque victorienne. Alors qu'au milieu du siècle, l'attention se portait encore prioritairement sur des villes industrielles comme Manchester, porteuses des craintes des uns et des espoirs révolutionnaires des autres ${ }^{57}$, désormais c'était l'orgueilleuse capitale impériale et financière qui symbolisait tous les risques et dysfonctionnements du gigantisme urbain $^{58}$. Stedman Jones a relevé que lorsque le terme "unemployment " fit son apparition dans la langue courante pendant les années 1880 , il était régulièrement associé à Londres ${ }^{59}$. On sait également l'impact qu'eurent les conflits sociaux de la métropole sur l'évolution du syndicalisme et du socialisme britanniques. Par ailleurs, des campagnes de presse ${ }^{60}$ avaient mis au premier plan les conditions de vie misérables qui prévalaient dans l'East End, ou la dépravation qui régnait dans la ville la plus peuplée au monde ${ }^{61}$. Des enquêtes plus rigoureuses, comme la monumentale enquête de Charles Booth, The Life and Labour of the People of London, publiée en 17 volumes entre 1891 et 1903, allaient confirmer ce constat accablant, et renforcer l'idée largement répandue que Londres était un " cas à part ", un cancer au milieu d'une nation saine. Il faudrait attendre 1901 et la publication des études de Rowntree sur la pauvreté à York pour accréditer l'idée que les petites villes de province n'étaient pas exemptes des maux de la capitale.

Tout autant que l'expression du communisme de leur auteur, le choix de certains termes par Morris est le reflet des inquiétudes de son époque. C'est le cas de la métaphore du jeu, utilisée de manière récurrente soit pour désigner les activités financières de la City, soit le capitalisme dans son ensemble ${ }^{62}$. En cette fin de siècle, l'essor du capitalisme boursier inquiète les tenants du capitalisme familial qui avait prospéré pendant la révolution industrielle. En 1890, alors qu'une Ligue nationale 
contre le jeu se constitue, un énorme scandale financier secoue la place de Londres ${ }^{63}$, ravivant les attaques contre l'« économie de casino». De nombreux articles fustigent les spéculateurs, assimilés aux parieurs et autres joueurs de baccara. L'amalgame déjà ancien entre ces activités sera l'un des principaux thèmes de certaines ligues de vertu jusqu'au début du vingtième siècle.

La théorie de la dégénérescence urbaine a déjà été évoquée, et de nombreux réformateurs prônaient, sous des formes diverses, l'établissement de "colonies agricoles » (farm colonies) pour arracher le « résidu » de la population des grandes villes à son environnement délétère. C'était notamment le cas du chanoine Barnett, qui avait déjà lancé le mouvement des "settlements" dans l'East End ${ }^{64}$. Un projet analogue était défendu par le fondateur de l'Armée du Salut, le "général » William Booth, dans un ouvrage ${ }^{65}$ publié la même année que Nouvelles de Nulle Part. De tels projets étaient trop réformistes pour recevoir l'assentiment de Morris. Il y voyait de simples palliatifs là où une transformation radicale de la société s'imposait. Son propos était plus sévère pour l'Armée du Salut, accusée d'enrégimenter les pauvres ${ }^{66}$. Il est vrai que la vision de Morris se distinguait par ses aspects libertaires, sur la sexualité par exemple, ou encore sur la répression de la criminalité. À bien des égards, il se heurtait de front aux conventions victoriennes.

Néanmoins, s'il tenait son siècle en horreur, Morris en exprimait fort bien certains goûts et dégoûts. La détestation de la grande ville, cloaque de misère et source de subversion, s'inscrit parfaitement dans le discours dominant de l'époque.

\section{Les grands inspirateurs}

Dans ses considérations sur les rapports entre ville et campagne, Morris fait parfois écho aux préoccupations de son époque, mais il s'appuie également sur une tradition plus ancienne. À la fin de Nouvelles de Nulle Part, le narrateur se retrouve brutalement en 1890 et croise un ouvrier agricole servile et prématurément vieilli. En brossant le portrait de cette victime, Morris reprend l'un des thèmes de ses écrits journalistiques et conférences : la dénonciation d'une Angleterre rurale défigurée par les fausses valeurs urbaines et exploitée par le capitalisme agraire.

31 Mais le sentiment de perte d'une Arcadie menacée par les valeurs de la ville était déjà largement exprimé au XVIII ${ }^{\mathrm{e}}$ siècle. Le triste sort du vieil ouvrier agricole, figure inversée de la pastorale, est ainsi évoqué par Crabbe dans son célèbre poème, The Village (1783). La disparition d'un ordre rural ancien souvent idéalisé est également déplorée dans la poésie de Goldsmith et John Clare ${ }^{67}$. Quant au vieux stéréotype moralisateur opposant vertu rurale et vice urbain, réactualisé par la société de consommation, il imprègne La Tâche, de Cowper (1785). La diabolisation par Morris de la civilisation industrielle et de Londres renvoie aux 'satanic mills' flétris par Blake et à certains vers de Keats et Shelley ${ }^{68}$. On pourrait multiplier les exemples et les prolonger dans la littérature romanesque du XIX ${ }^{e}$ siècle $^{69}$.

La dette envers Thomas More est plus ouvertement revendiquée, ne serait-ce qu'à travers le titre ${ }^{70}$ de Nouvelles de Nulle Part, et à plusieurs reprises, Morris affirmera que l' Utopie « doit nécessairement figurer dans une bibliothèque socialiste ${ }^{71}$. Si l'utopie de Morris présente autant de dissemblances que de ressemblances avec celles de More, il convient de souligner la dénonciation par ce dernier des effets dévastateurs de la politique des clôtures sur les paysans, arrachés à leurs demeures et souvent voués au 
brigandage. Le parallèle est aisé à établir avec la fin du dix-neuvième siècle, marquée, comme on l'a vu, par le dépeuplement des campagnes et par la chute brutale de la culture du blé, victime de la concurrence étrangère, au profit de l'élevage ${ }^{72}$. D'autres similitudes sont observables dans les sociétés imaginées par les deux auteurs : alors que dans l'Angleterre du XVI ${ }^{e}$ siècle, l'agriculture est sacrifiée au profit de l'élevage et du commerce de la laine, sa prééminence est rétablie dans l'utopie de More :

Il est un art commun à tous les Utopiens, hommes et femmes, et dont personne n'a le droit de s'exempter, c'est l'agriculture. Les enfants l'apprennent en théorie dans les écoles, en pratique dans les campagnes voisines de la ville, où ils sont conduits en promenades récréatives. Là, ils voient travailler, ils travaillent eux-mêmes, et cet exercice a de plus l'avantage de développer leurs forces physiques ${ }^{73}$.

Un autre texte exerça une forte influence sur Morris, il s'agit d'After London, roman d'anticipation publié en 1885 par Richard Jefferies ${ }^{74}$. Détruite par un cataclysme, l'Angleterre est retournée à la barbarie, des tribus s'y affrontent et l'organisation sociale est fondée sur l'esclavage. Les villes, comme le goût de la culture et des livres, ont disparu et les rescapés ont été disséminés dans les campagnes. Cette terre dévastée où règne la dégradation morale est bien différente de l'Angleterre de Nouvelles de Nulle Part, elle contient toutefois des germes annonciateurs d'épanouissement humain. Y est également décrit l'envahissement de l'Angleterre dépeuplée par une végétation luxuriante. Ne subsistent que forêts et marécages, et ce triomphe de l'élément végétal se retrouve, sous une forme très atténuée, dans de nombreux écrits de Morris. A défaut de voir, comme dans sa fiction, la forêt envahir la ville, il exprime dans la presse le vœu qu'on cesse d'étêter et d'émonder les arbres de Londres afin que la végétation dissimule le plus possible les hideurs de la ville ${ }^{75}$.

De telles vues n'étaient pas entièrement nouvelles : aux dix-septième et dix-huitième siècles, des voix s'étaient déjà élevées pour réclamer qu'une végétation plus abondante et moins entravée donne à Londres l'aspect d'une cité rurale, voire d'une cité forestière ${ }^{76}$. Certes, à travers son plaidoyer pour l'intégrité des arbres, à la ville comme à la campagne, Morris s'en prend à deux siècles d'influence néfaste sur le paysage britannique, celle des partisans de l'improvement, des paysagistes adeptes d'un naturel factice comme Capability Brown ${ }^{77}$, ou encore des créateurs d'un bocage adapté aux besoins de la chasse au renard; mais, pour ce qui est de l'entretien des arbres proprement dit, les vues de ceux qu'il critiquait étaient parfois proches des siennes. En revanche, d'autres ont défendu dans l'usage du sécateur l'expression d'une conception rigoriste de l'éducation et de la société ${ }^{78}$. Au-delà du parti pris esthétique, les diatribes récurrentes de Morris contre l'émondage et l'étêtage peuvent plutôt se replacer dans une tradition qui voit dans la libre croissance de l'arbre le symbole des libertés anglaises, liberté des mœurs tout autant que libertés politiques.

On connaît l'influence de Carlyle et de Ruskin sur la pensée de Morris, notamment avant la conversion de ce dernier au socialisme en 1883. C'est en lisant Past and Present pendant ses études à Oxford que le jeune homme avait trouvé pour la première fois la dénonciation de la société bourgeoise et industrielle. Comme beaucoup, il fut marqué par les formules fulgurantes du penseur de Cheyne Walk, qui dénonçait le 'cash nexus' d'une société régie par l'argent tout comme « la cauchemardesque vulgarité » (Cockney nightmare) d'un univers gouverné par le Whiggisme et ses dogmes économiques. Le fervent lecteur de Scott qu'était Morris fut également sensible au médiévisme de Carlyle dont certains écrits prônaient le retour à la nature, mais la dérive du vieux maitre vers un autoritarisme réactionnaire exclut toute influence véritable sur le 
militant de 1890. Le lien avec Ruskin est plus palpable, malgré la divergence politique. Nous retiendrons surtout l'hostilité à la ville et au machinisme qui s'exprime dans les écrits de ce dernier. Ainsi rêve-t-il dans The Crown of Wild Olive (1866) d'une Angleterre retournée à l'agriculture et au travail manuel. Le même ouvrage s'ouvre sur une protestation amère contre les transformations qui ont souillé la campagne aux bords de la rivière Wandle. Quant à l'horreur du pullulement urbain, elle s'exprime avec plus de virulence encore dans Fors Clavigera, série épistolaire publiée entre 1871 et 1884 : ces "horribles nids, que vous appelez des villes, ne sont guère plus que des laboratoires pour la distillation dans le ciel de fumées et d'odeurs vénéneuses, mêlées aux effluves de matière animale pourrissante et aux miasmes infectieux de maladies purulentes ${ }^{79}$.

On a pu parler de «socialisme médiéval » au sujet de Ruskin et sa dénonciation de la déshumanisation engendrée par la division du travail a eu un impact indéniable sur des futurs militants socialistes et travaillistes. Certaines expressions qu'il utilise, comme par exemple, la comparaison des injustices du " pouvoir morbide de l'industrie et du commerce " avec " celles d'une maison de jeu " ${ }^{80}$, ont été reprises dans Nouvelles de Nulle Part pour stigmatiser les activités de la City, comme nous l'avons vu. En revanche, Morris n'a jamais adhéré à sa conception idéaliste et hiérarchisée de la société ${ }^{11}$. Un écart politique tout aussi important le séparait de William Cobbett, radical venu du toryisme animé par la haine de l'utilitarisme et du « laissez-faire ». Cela n'empêcha pas Morris, admirateur et lecteur fervent de Rural Rides, de s'inspirer largement de son impétueuse réhabilitation du Moyen Âge. Pour Cobbett, le parasitisme et la concentration urbaine ont éloigné les hommes de ce sain "état de nature » qui subsistait au Moyen Âge. À ses yeux, la croissance des grandes villes est monstrueuse et il utilise le terme méprisant de "Verrue » (the Wen), repris par Morris, pour désigner Londres. Cobbett et Morris partagent de nombreuses indignations, où l'émondage des arbres figure en bonne place. Il apparaît donc qu'à travers son amour de la campagne anglaise et de ses paysages, Morris, malgré son athéisme et son adhésion au matérialisme historique, puise à de nombreuses sources, y compris conservatrices.

\section{Postérité du ruralisme de William Morris}

S'il est une question sur laquelle William Morris semble avoir fait des émules, c'est celle de la densité de l'habitat. On se souvient que dans Nouvelles de Nulle Part, cela donne lieu à un débat animé avec le père d'Ellen, l'hôte d'un soir lors de l'étape de Runnymede ${ }^{82}$. A plusieurs reprises, on observe que l'avènement d'une société socialiste a donné lieu à une dispersion de l'habitat ${ }^{83}$. Ici encore, la fiction rejoint les vues exposées par Morris dans ses articles et conférences. Quand en 1884, il réagit à la création de la Royal Commission on the Housing of the Working Classes, il envisage l'existence d'immeubles collectifs dans des « rues verticales », mais c'est afin de permettre la création de grands jardins accessibles à tous. En outre, une telle solution ne saurait être que provisoire puisque liée à l'existence de grandes villes, temporaire à ses yeux ${ }^{84}$. La ville qu'il appelle de ses vœux ('what a city might be'), décrite dans une conférence prononcée à la fin de sa vie (Makeshift, 1894), présente davantage de similitude avec le concept de cité-jardin qui sera développé plus tard par Ebenezer Howard ${ }^{85}$. Sa structure concentrique, qui s'organise autour de grands édifices publics, comprend une 'suburb' qui ressemble plus à une ceinture verte qu'à une banlieue proprement dite. Les propos tenus dans une autre conférence vont dans le même sens : 
I want neither the towns to be the appendage of the country, nor the country of the town; I want the town to be impregnated with the beauty of the country, and the country with the intelligence and the vivid life of the town... In short, [I want the town to be] a garden with beautiful houses in it. ${ }^{86}$ situées aux environs de Londres, l'une à Letchworth, l'autre à Welvyn. Aucun des deux projets ne connut le succès escompté, mais le mouvement des cités-jardins a toutefois joué un rôle déterminant dans la discipline naissante de l'urbanisme. L'idée initiale de contenir la croissance des grandes villes grâce à une constellation de cités-jardins autonomes s'est diluée au profit de la notion moins ambitieuse de banlieue-jardin, mise en oeuvre à Hampstead. Elle est surtout à l'origine de la création des villes nouvelles et des ceintures vertes. A défaut de la rupture radicale attendue par le révolutionnaire qu'était Morris, les mesures palliatives dont il se méfiait semblent avoir prévalu. Le réformiste qu'était Howard ne cite jamais Morris, mais il convient de signaler la présence de Raymond Unwin parmi les jeunes sympathisants de la Socialist League qui venaient rencontrer le vieux sage d'Hammersmith ${ }^{87}$. Il n'est donc pas absurde d'inclure Morris dans la matrice intellectuelle de l'aménagement du territoire britannique, dont Unwin fut l'un des principaux acteurs.

que l'étalement urbain a perduré au vingtième siècle. L'expansion de la vulgarité 'cockney' dénoncée par Morris fut particulièrement marquée dans l'entredeux-guerres. Les difficultés financières de l'État et le développement anarchique de lotissements périurbains souvent confiés à des investisseurs privés se traduisirent par une version parfois caricaturale de l'habitat à faible densité prévu par les nouvelles réglementations. George Orwell a dressé un tableau accablant de ces ribbondevelopments des années trente ${ }^{88}$. L'urbanisation incontrôlée des campagnes est à l'origine du Council for the Preservation of Rural England (CPRE), fondé en 1926, et dont un autre cheval de bataille était la défiguration du paysage par les panneaux publicitaires. Quand on sait que le dernier discours prononcé par Morris le fut devant la Society for Checking the Abuses of Public Advertising ${ }^{89}$, la tentation est grande d'établir une filiation entre le CPRE et le fondateur de l'Anti-scrape, qui s'était résigné à accepter le principe de l'action parlementaire ${ }^{90}$. Certes, le très conservateur CPRE était aux antipodes des ambitions révolutionnaires de l'ancienne Socialist League, mais cette proximité de surface montre bien que le combat pour la préservation des paysages peut osciller entre nostalgie, voire élitisme, et contestation radicale.

Quel fut l'écho de ce "ruralisme » de Morris dans la gauche britannique? Sans doute le trouve-t-on dans la nébuleuse de mouvements sportifs et culturels qui gravitaient autour de Robert Blatchford et de sa revue, The Clarion. Dans son best-seller publié en 1894, Merrie England ${ }^{91}$, il reprend de nombreux thèmes chers à Morris, notamment dans le chapitre 'Town $v$. Country' et il est bien connu que des générations de militants du premier vingtième siècle ont été imprégnées du culte du grand air et du goût des grands espaces. En revanche, la réalité électorale de l'entre-deux-guerres amena le parti travailliste à inverser cette appréciation classique de la ville et de la campagne. Autrefois synonyme de déclin, la ville semblait représenter une avancée vers une société plus libre et plus civilisée. Il reste que ce discours imposé par le contexte politique était en décalage avec un héritage culturel prégnant ${ }^{92}$. Ce décalage a toujours été une source de malaise, non seulement pour le Parti travailliste, mais pour la gauche britannique dans son ensemble.

Revue Française de Civilisation Britannique, XIII-1 | 2004 


\section{NOTES}

1. Karl MARX \& Friedrich ENGELS, Manifest der Kommunistischen Partei, London: Gedruckt in der Office der 'Bildungs-Gesellschaft für Arbeiter' von J.E. Burghard, 1848 (Manifeste du parti communiste [première traduction française, 1893], Paris : Éditions sociales, 1976, p. 57).

2. Ibid, p. 36: «La bourgeoisie a soumis la campagne à la domination de la ville. Elle a créé d'énormes cités; elle a prodigieusement augmenté les chiffres de population des villes par rapport à la campagne, et, par là, elle a arraché une partie importante de la population à l'abrutissement de la vie des champs. » C'est nous qui soulignons.

3. On aura compris que ce narrateur ressemble étrangement à l'auteur lui-même, qui n'en fait aucun mystère (âge qu'il décline, prénom qu'il se donne, etc.).

4. Rappelons que Nouvelles de Nulle Part fut publié en feuilleton dans Commonweal, l'organe de la Socialist League, en 1890 avant de faire l'objet d'une publication séparée et enrichie en 1891.

5. On observera la continuité thématique avec le prologue de The Earthly Paradise, le recueil de poèmes dont la publication en 1868 avait assis la réputation de Morris : 'Forget the spreading of the hideous town, Think rather of the pack-horse on the down, And dream of London, small, and white, and clean, The clear Thames bordered by its garden green'.

6. 'Amidst all these gardens and houses it was of course impossible to trace the site of the old streets, but it seemed to me that the main road ways were the same as of old... From the southern side of the said orchard ran a long road... at the end of which showed the high tower of the Parliament House, or Dung Market.' p. 77, in William MORRIS, News from Nowhere and Other Writings, Clive WILMER (ed.), London: Penguin Books, 1998.

Ibid, p. 61: 'For King Street was gone, and the highway ran through wide sunny meadows.'

Ibid, p. 84: 'We went down on a gentle slope through some beautiful rose-gardens, laid out on what I took to be the site of Endell Street.'

7. News from Nowhere, p. 100 : 'But in the city we have a thickly-dwelling population... therefore it remains the most populous part of London, or perhaps of all these islands.'

8. Ibid, p. 99.

9. Ibid, p. 102.

10. Ibid: 'The big murky places which were once the centres of manufacture... have left less signs of their existence than London. Ibid, p. 140 : 'At a place called Manchester, which has now disappeared.'

11. Ibid, p. 84 .

12. Ibid: 'There are the towns still with their streets and squares and market-places; so that it is by means of these smaller towns that we of to-day can get some kind of idea of what the towns of the older world were like ; - I mean to say at their best.'

13. Ibid, p. 103 : 'Yet there are many towns which have become scarcely less beautiful.' Voir aussi les propos de Dick sur la reconstruction de Reading. Ibid, p.187.

14. Ibid., p. 61.

15. Town and Country, conférence donnée le 29 mai 1892. Larges extraits reproduits sur : <http://www.marxists.org/archive/morris/works/tmp/town.htm>

La Revue française de civilisation britannique remercie Shannon L. ROGERS, Editor, Newsletter of the William Morris Society in the United States, de sa précieuse collaboration pour la datation de certaines conférences, dont Town and Country.

16. Morris avait effectué le même trajet en barque. En outre, Kelmscott Manor, sa propriété aux abords des sources de la Tamise, présente des similitudes avec le point d'arrivée.

17. News from Nowhere, p. 183 et p. 186 : '... what success the new order of things had had in getting rid of the sprawling mess... about Reading and Caversham.'

18. Ibid, pp. 55-56. 
19. Voir Marion SHOARD, A Right to Roam, Oxford: Oxford University Press, 1999, pp. 159-161. Le contentieux sur la forêt de Berkhamstead, au nord-ouest de Londres, donna également lieu à des violences.

20. Celle-ci figurait parmi les nombreuses associations auxquelles, bien avant son adhésion au socialisme, Morris ne ménageait pas son soutien. Voir E.P. THOMPSON, William Morris : Romantic to Revolutionary, London: Lawrence \& Wishart, 1955 (Stanford: Stanford University Press, 1976, p. 257).

21. Un common, terme souvent traduit par «communal », est le plus souvent un terrain privé, mais grevé de droits de servitude (libre accès du public, collecte de bois, glanage, vaine pâture, etc.).

22. News from Nowhere, p. 194. D'une façon inattendue, mais qui confirme son animosité, il mentionne encore cette profession quand il s'agit de fustiger l'étêtage des arbres. Ibid, p. 183: 'The banks of the forest... had lost their courtly gamekeeperish trimness.'

23. L'opinion de Morris sur la battue transparaît dans l'usage métaphorique du terme qu'il fait en 1884 pour attaquer la politique étrangère britannique. Il qualifie les massacres de paysans égyptiens de «battue pour le compte des usuriers et détenteurs d'obligations »: 'The Bondholders Battue', Justice, Vol. 1, n 4, 9 February 1884. Reproduit dans William MORRIS, Political Writings : Contributions to Justice and Commonweal 1883-1890. Edited by Nicholas SALMON. Bristol: Thoemmes, 1994, pp. 10-13.

24. Voir Emmanuel ROUDAUT, «Les Hordes urbaines dans nos campagnes : la longue marche des randonneurs", pp. 225-241 in Emmanuel ROUDAUT (ed.), Villes et campagnes britanniques: confrontation ou (con)fusion?, Valenciennes : Presses Universitaires de Valenciennes, 2003.

25. Gareth STEDMAN JONES, Outcast London : A Study in the Relationship between Classes in Victorian Society, Oxford: Clarendon Press, 1971 (London : Penguin , 1992, pp. 127-151).

26. News from Nowhere, p. 104.

27. Il y en aura d'autres, par exemple sur l'absence de scolarisation des enfants, de notion de valeur marchande, de propriété privée, etc.

28. News from Nowhere, p. 63.

29. La proximité des analyses de Marx, Engels et Morris sur ce point est établie. Voir Paul MEIER, La Pensée utopique de William Morris, Paris : Éditions sociales, 1972, p. 611.

30. William MORRIS, News from Nowhere, p. 104, cité par MEIER, op. cit., p. 613.

31. Ibid., également cité par MEIER.

32. News from Nowhere, p. 177.

33. On notera la différence entre l'aspect physique des héroïnes de Nouvelles de Nulle Part et les visages diaphanes que l'on trouve dans les tableaux préraphaélites. Sur ce point, voir entre autres Paul MEIER, La Pensée utopique de William Morris, p. 147 et p. 638.

34. 'The leisurely, but not stupid, country-life which grew to be common amongst us', News from Nowhere, p. 160.

35. Ibid., p. 193.

36. Ibid., p. 105.

37. Makeshift, conférence donnée le 18 novembre 1894. Reproduite sur :

<http://www.marxists.org/archive/morris/works/tmp/make.htm>

38. News from Nowhere, p. 219.

39. Il convient de signaler la réhabilitation de fêtes comme celles des moissons dans une perspective opposée à celle de Morris. Au milieu du XIX ${ }^{\mathrm{e}}$ siècle, on peut parler de « réinvention de cette tradition », canalisée par le clergé et les élites locales pour en faire une démonstration de patriotisme et de piété.

40. Town and Country, loc. cit.

41. News from Nowhere, p. 118.

42. 'The last harvest, the last baby ...' (ibid., p. 89), voir également ibid., p. 224. 
43. William MORRIS, 'Looking Backward', Commonweal, vol. 5 , nº180 (22 juin 1889). Reproduit dans William MORRIS, Political Writings : Contributions to Justice and Commonweal 1883-1890, p. 423.

44. Paul MEIER, La Pensée utopique de William Morris, pp. 614-615.

45. Ibid., pp. 602-603.

46. News from Nowhere, p. 70 : 'Here was the ghost of London still asserting itself as a centre - an intellectual centre, for aught I knew.' Notons que Londres n'est plus qu' « un fantôme ", l'ombre d'elle-même et que cette prétention fait sourire le narrateur.

47. Ibid., p. 68. 'I don't think we need fear having too many book-learned men... After all, I don't know that it does them much harm, even if they do grow up book-students'. Voir également les propos de Dick sur le goût de son aïeul pour les lives et l'histoire (ibid., p.175). Voir aussi ibid., p. 94 : 'I am a "literary man", as we queer animals used to be called'.

48. 'Under an Elm-tree; or thoughts in the Countryside', Commonweal, vol. 5, nº182 (6 juillet 1889). Reproduit dans William MORRIS, Political Writings: Contributions to Justice and Commonweal $1883-1890$, p. 430.

49. Socialism, its Growth and Outcome, pp. 313-316, cité par Paul MEIER, La Pensée utopique de William Morris, pp. 604-605.

50. Ibid., p. 612.

51. Ibid., p. 615.

52. News from Nowhere, p. 119.

53. Adam BUICK, 'William Morris and Incomplete Communism: A Critique of Paul Meier's Thesis', Journal of the William Morris Society, vol. 3, n², 1975, pp. 16-32.

54. Si d'aventure, ils venaient à subsister, ils seraient morcelés en unités autonomes (wards).

55. 'Ugly London', Pall Mall Gazette, 4 September 1888. Article reproduit sur :

<http://www.marxists.org/archive/morris/works/1888/ugly.htm>

56. Town and Country, loc. cit.

57. Voir notamment l'ouvrage de Friedrich ENGELS, Die Lage der arbeitenden Klasse in England, Leipzig, 1845 (La Situation de la classe laborieuse en Angleterre). L'exposition universelle de 1851 a bien entendu joué un rôle (positif celui-là) dans le déplacement d'intérêt vers Londres.

58. Voir Alun HOWKINS, 'The Discovery of Rural England', in Robert COLLS \& Philip DODD (eds.), Englishness, Politics and Culture, 1880-1920, London: Croom Helm, 1986, pp. $62-88$ (p. 65).

59. Outcast London, op. cit., p. xxv (préface de la première édition).

60. L'une d'entre elles était liée aux écrits d'un pasteur sur sa paroisse de l'East End (Andrew MEARNS, The Bitter Cry of Outcast London, London: James Clarke \& Co. 1883). Leur retentissement se traduisit notamment par la création d'une commission d'enquête (Royal Commission on the Housing of the Working Classes, 1884).

61. Lorsqu'il utilise l'expression 'Modern Babylon' dans News from Nowhere (op.cit., p. 99), Morris fait une allusion transparente à une série d'articles dénonçant la prostitution et le trafic de jeunes filles, publiée en 1885 dans la Pall Mall Gazette sous le titre de 'The Maiden Tribute of Modern Babylon'. Série reproduite sur :

<http://www.attackingthedevil.co.uk/pmg/tribute/>

Morris avait prononcé le 5 août 1885 un discours sur ce scandale en s'abstenant de porter un jugement moral sur les individus tout en soulignant les effets dégradants du capitalisme : 'Speech on W.T. Stead's Exposé of London Prostitution', reproduit sur :

<http://www.marxists.org/archive/morris/works/tmp/stead.htm>

Parmi les scandales ayant l'East End pour cadre, citons également l'affaire « Jack l'éventreur », en 1888.

62. Voir par exemple News from Nowhere, pp. 100 et 110.

63. La banque Baring Brothers, déjà !

64. 'A Scheme for the Unemployed', Nineteenth Century, vol. 24 (November 1888), pp. 753-754, cité par STEDMAN JONES, Outcast London, p. 304. 
65. William BOOTH, In Darkest England and the Way Out, London: Salvation Army, 1890. Une expérience fut menée sur un terrain acquis par l'Armée du Salut à Hadleigh, dans l'Essex.

66. 'Workhouse Socialism', Commonweal, Vol. 6, $\mathrm{n}^{\circ} 251$ (1 November 1890). Reproduit dans William MORRIS, Political Writings : Contributions to Justice and Commonweal 1883-1890, pp. 484-487.

67. Oliver GOLDSMITH, The Deserted Village, London, 1770. John CLARE, Poems Descriptive of Rural Life and Scenery, 1820. Voir Raymond WILLIAMS, The Country and the City, Oxford: Oxford University Press, 1973 (un classique) et Jacques CARRÉ, « L'Invention du patrimoine rural », in E. ROUDAUT, op. cit., pp. 21-35.

68. 'Attempts to make Hell happy, instead of a resolution to get rid of it' ('Ugly London', loc. cit.). 'Hell is a city much like London/ A populous and a smoky city' (SHELLEY, 'Peter Bell the Third', 1819).

69. Paul MEIER (La Pensée utopique de William Morris, p. 609) a ainsi relevé chez Morris de nombreuses réminiscences du chapitre IV du Barnaby Rudge de Dickens, où s'exprime la nostalgie du Londres verdoyant de l'époque préindustrielle.

70. Autre référence manifeste dans ce titre, celle à Samuel BUTLER (Erewhon, 1872).

71. Paul MEIER, La Pensée utopique de William Morris, p. 93.

72. Pour la période $1867-71$, le blé représentait $20 \%$ du produit agricole brut ; en 1894-98, sa part était tombée à $7 \%$. Entre 1878 et 1894 , la superficie des terres consacrées à sa culture chuta de 40\% (G.E. MINGAY, Land and Society in England, 1750-1980, London: Longman, 1994, pp. 202-204).

73. Thomas MORE, L'Utopie, [1516] (Avec introduction et notes de Marcelle Bottigelli-Tisserand. Paris : Éditions sociales, 1976, p. 124). Voir également pp. 81-83.

74. Voir Paul MEIER, La Pensée utopique de William Morris, pp. 107-114 et 353-354.

75. 'Ugly London', loc. cit.

76. John EVELYN, Fumifugium, 1661 ; Thomas FAIRCHILD, The City Gardener, 1722 ; Batty LANGLEY, A Sure Method of Improving Estates by Plantation, 1728 ; Jonas HANWAY, Letters on the Importance of the Rising Generation, 1767. Voir Keith THOMAS, Man and the Natural World, London: Allen Lane, 1983 (Harmondsworth: Penguin, 1984, pp.198-223).

77. Des villages avaient parfois été rayés de la carte pour faire place à des parcs d'agrément autour des demeures aristocratiques.

78. 'The luxuriancy and vigour of most healthful trees is like the extravagant sallies of youth, who are apt to live too fast, if not kept within due bounds and restrained by seasonable corrections. [Regular pruning keeps] all in order, which would otherwise be perfect anarchy and confusion' (John LAURENCE, New System of Agriculture, 1726, cité par Keith THOMAS, op. cit., p. 221).

79. Fors Clavigera, Letters to the Workmen and Labourers of Great Britain, lettre 5, § 15, 16 (traduction de Paul MEIER, La Pensée utopique de William Morris, p. 191).

80. Ibid., lettre 15 (traduction de Paul MEIER, La Pensée utopique de William Morris, p. 223).

81. Il est significatif qu'il se soit tenu à l'écart de la Guilde de Saint Georges, expérience utopique lancée par Ruskin en 1871.

82. News from Nowhere, p. 180.

83. Ibid., p. 106.

84. 'It might be advisable, granting the existence of huge towns for the present, that the houses for workers should be built in tall blocks', 'The Housing of the Poor', Justice, Vol. 1, n`27, 19 July 1884. Reproduit dans William MORRIS, Political Writings : Contributions to Justice and Commonweal 1883-1890, p. 51.

85. Ebenezer HOWARD, Tomorrow: a Peaceful Path to Real Reform, 1898 (réédité en 1902 sous le titre de Garden Cities of Tomorrow).

86. Town and Country, loc.cit.

87. Voir E.P. THOMPSON, William Morris : Romantic to Revolutionary, p. 555.

88. George ORWELL, Coming Up for Air, London: Victor Gollancz, 1939. Le même phénomène suscitait toujours l'indignation de Morris au soir de sa vie (E.P. THOMPSON, William Morris: Romantic to Revolutionary, p. 633). Voir également G.E. CHERRY, Town Planning in Britain, Oxford: Blackwell, 1996. 
89. E.P. THOMPSON, William Morris : Romantic to Revolutionary, p. 630.

90. Ce dernier discours, où il se déclare en faveur d'une proposition de loi, en est un des nombreux signes.

91. Robert BLATCHFORD, Merrie England [1894], New York: Monthly Review Press, 1966.

92. Voir Clare GRIFFITHS, «'Ville' et 'campagne'dans la rhétorique politique britannique entre les deux guerres ", in E. ROUDAUT, op. cit., pp. 73-89.

\section{RÉSUMÉS}

Dans News from Nowhere, la mégalopole victorienne qu'était Londres a cédé la place à des bourgades éparpillées dans la forêt, à tel point que le vœu exprimé par Marx et Engels de voir se dissoudre l'opposition entre ville et campagne semble réalisé. Instruisant le procès du capitalisme et des souillures de la civilisation industrielle, cette œuvre présente une société communiste où la grande ville a disparu. Le caractère idyllique de ces représentations reste toutefois très en phase avec certaines valeurs de la société qu'elles dénoncent. On tentera ici de cerner les contours d'un ruralisme aussi dominant sur le plan culturel que politiquement ambigu.

In News from Nowhere, the urban giant that was Victorian London has given way to a semi-rural built-up environment scattered in the forest, to such an extent that Marx and Engels's aim to bridge the gap between town and country seems to have been reached. An indictment of capitalism and of the horrors of industrialisation, Morris's "romance" presents a communist society in which large cities have disappeared. However, such an idyllic picture conveys some of the very values of the society it claims to denounce. This paper will examine the cultural and social context of a clearly anti-urban and pro-rural stance, and attempt to assess its political ambivalence.

\section{AUTEUR}

\section{EMMANUEL ROUDAUT}

Université de Valenciennes 